\title{
Tamoxifen for Recurrent High-Grade Glioma: A Retrospective Study
}

\author{
De Roxas $R C^{*}$, Omene $E$, Young $K$, Coppens R, Fulton DS and Easaw JC \\ Cross Cancer Institute, University of Alberta, Edmonton, Canada
}

\begin{abstract}
Introduction: The management of recurrence in patients with high-grade glioma remains controversial. The lack of clear molecular targets to guide systemic therapy for patients has resulted in the use of various systemic agents with modest efficacy but with significant side effects. Tamoxifen has been used in our centre with some success and excellent tolerability.

Methodology: We retrospectively collected clinical data of patients aged 18-65 years with recurrent high-grade glioma from 2003-2016. A total of 167 patients were identified and their demographic, clinicopathologic and follow-up data were retrieved. All patients received tamoxifen as part of their chemotherapeutic regimen. Univariate data analysis was utilized in the study.

Results: All patients included in the study underwent initial surgery, radiotherapy and experienced disease recurrence. They were typically heavily pretreated with approximately $60 \%$ of the patients receiving at least three chemotherapeutic agents before starting tamoxifen. We observed that patients on tamoxifen had a median overall survival of 24.9 weeks (range: 1.6 to 346.1 weeks). Almost all patients (80\%) of patients progressed on tamoxifen. We observed no statistical difference in median overall survival and time to progression between glioblastoma and non-glioblastoma patients treated with tamoxifen. Importantly, tamoxifen was not associated with significant toxicity.

Conclusion: This study demonstrates survival data on par with historical data of other chemotherapeutic agents for those with progressive disease and even longer median overall survival in those who are able to achieve stable disease or partial response. Single-agent, oral tamoxifen is an appealing target for the heavily pre-treated patient population of recurrent high-grade glioma and further studies should be explored.
\end{abstract}

\section{Introduction}

High-grade gliomas include both glioblastoma multiforme (GBM) and grade 3 (anaplastic) astrocytoma and oligodendroglioma. The management of recurrence in patients with high-grade glioma remains controversial. Currently, three approaches to management include repeat surgery, re-irradiation, and systemic therapy used either alone or in combination [1]. Depending on local expertise and preference, re-irradiation and repeat surgery are used variably in the management of recurrence but in almost all cases, some sort of systemic therapy is attempted. Regardless of the treatment modality employed, survival for these patients is often dismal and varies between 6 to 15 months $[2,3]$.

Despite advances in molecular diagnostic approaches incorporating predictive markers such as 06-methylguanine-DNA methyltransferase (MGMT) promoter methylation and $1 p / 19 q$ co-deletion to guide first-line therapy of high-grade glioma, we lack clear molecular targets to guide systemic therapy used in recurrence. As a result, a standard of care for recurrent or progressive disease has not been established, leading to the use of a variety of non-targeted sys- temic agents. Commonly used agents in recurrence include temozolomide rechallenge and lomustine (CCNU) $[4,5]$; however, other agents including etoposide, bevacizumab, irinotecan, cis-retinoic acid and other nitrosoureas, either as a single agent or in combination have also been explored [6-9]. Data from a retrospective review and a meta-analysis evaluating various systemic agents for recurrent high-grade glioma show median overall survival (mOS) rates which vary between 28 and 44 weeks $[10,11]$.

In addition to improving survival, a key factor guiding the choice of systemic agent for recurrent high-grade glioma is the minimization of side effects. One drug that has been

*Corresponding author: Dr. De Roxas RC, Cross Cancer Institute, University of Alberta, Edmonton, AB T6G 1Z2, Canada

Accepted: July 29, 2020

Published online: July 31, 2020

Citation: De Roxas RC, Omene E, Young K, et al. (2020) Tamoxifen for Recurrent High-Grade Glioma: A Retrospective Study. Insights Neurooncol 3(1):43-47 
used with some success and excellent tolerability has been tamoxifen. Tamoxifen is an oral non-steroidal anti-estrogen compound whose effectiveness in treating hormone receptor positive breast cancer is well established. In general, tamoxifen has only few and relatively mild acute side effects such as hot flashes, gastrointestinal disturbances, vaginal discharge, and menstrual irregularities in premenopausal women. More serious adverse effects such as blood clots and secondary hematologic malignancy are rare [12]. Tamoxifen's anti-neoplastic effect in high-grade gliomass have been shown to be pleiotropic, acting at sites and in pathways distinct from the estrogen receptor, including the inhibition of the protein kinase $\mathrm{C}$ and inhibition of the glutamine transporters in several in vitro studies [13-15].

Two single arm phase II trials assessed the benefit of single agent tamoxifen in high-grade glioma patients $[15,16]$. In one study, 20 patients with GBM and 12 patients with anaplastic astrocytoma experienced recurrence after treatment with radiation therapy and were given daily high-dose oral tamoxifen at a dose of $200 \mathrm{mg} /$ day for males and $160 \mathrm{mg} /$ day for females. The mOS for the GBM patients was 31 weeks from the time of recurrence while mOS for the anaplastic astrocytoma patients was 69 weeks [15]. A second study using single agent oral tamoxifen evaluated 24 patients with recurrentce of anaplastic astrocytoma after surgery and radiation therapy. A dose of $80 \mathrm{mg} / \mathrm{m}^{2}$ of tamoxifen was given orally either once daily or twice daily as salvage therapy and mOS was 52 weeks from the time of recurrence [16]. In both studies, toxicity due to tamoxifen was minimal.

Based on these reports, tamoxifen has been used for late stage recurrence in patients with high-grade glioma in our centre. In this retrospective study, we report the impact of tamoxifen on progression and survival.

\section{Methodology}

We retrospectively collected clinical data of patients treated at Cross Cancer Institute in Edmonton, Alberta, Canada which is tertiary treatment center for all patients diagnosed with glioma in northern Alberta (population 2.5 million). Patients aged 18 years and older with recurrent high-grade glioma after first-line treatment from January 2003 to December 2016 were included. One hundred sixty seven patients were identified and their demographic, clinicopathologic and follow-up data were retrieved. Two patients with insufficient data and inadequate follow-up were excluded. This study was approved by the local institutional ethics board.

All patients received tamoxifen as part of their chemother- apeutic regimen at a dose of $100 \mathrm{mg}$ twice daily. Response to treatment was defined using the response assessment in neuro-oncology (RANO) criteria. Progressive disease was defined as $>25 \%$ increase in the radiographic size of the lesion while partial response was defined as $>50 \%$ decrease in the radiographic size of the lesion. Stable diseases were those that cannot be classified as either progressive disease or partial response. Time to progression (TTP) was defined as the time from the first tamoxifen administration to the progression of the disease. The median overall survival (mOS) was defined as the time from first tamoxifen administration to the time of death. The results were tabulated using STATA 13. Univariate analysis using t-test for unequal variance was utilized to determine the significant difference between histologic groups.

\section{Results}

A total of 167 patients (96 males) aged 35 to 63 years (median age of 48.9 years) with high-grade glioma including transformed low-grade glioma $(n=52)$, recurrent anaplastic glioma $(n=34)$ and recurrent glioblastoma multiforme $(n=$ 81) were treated with tamoxifen. All patients underwent ini-

Table 1: Patient characteristics of enrolled patients.

\begin{tabular}{|l|l|}
\hline Age & Median ( $\mathbf{n}=167$ ) \\
\hline Sex & $\begin{array}{l}48.9 \text { (range: 35-63 } \\
\text { years) }\end{array}$ \\
\hline $\begin{array}{l}\text { Histology } \\
\text { Anaplastic Glioma }\end{array}$ & Male = 96 \\
\hline \multicolumn{1}{|c|}{ Anaplastic Astrocytoma } & \\
Anaplastic Oligoastrocytoma & 34 \\
\hline Glioblastoma multiforme & 23 \\
Low-grade glioma, transformed & 8 \\
\hline Adjuvant chemotherapy & 81 \\
Temozolomide & 52 \\
\hline Procarbazine-Lomustine-Vincristine (PCV) & 6 \\
\hline Lomustine (CCNU) & 90 \\
\hline Repeat Surgical resection & 2 \\
\hline Gross total re-resection & 12 \\
\hline Subtotal re-resection & 39 \\
\hline TTP & 10.8 weeks \\
\hline Incidence of thrombosis on tamoxifen & 7 (3 treated with \\
& anticoagulation) \\
\hline
\end{tabular}

Table 2: Median time to progression after tamoxifen chemotherapy between GBM vs. non-GBM patients.

\begin{tabular}{|c|c|c|c|c|}
\hline \multirow{2}{*}{ Response } & \multicolumn{4}{|c|}{ Median time to progression in weeks (\%) } \\
\hline & Non-GBM $n=86$ & p-value & GBM $\mathbf{n}=81$ & p-value \\
\hline Progressive disease & $9.7(79.8)$ & \multirow{2}{*}{$<0.001$} & $8.5(83.1)$ & \multirow{2}{*}{0.002} \\
\hline Stable disease & $34.4(17.7)$ & & $23.6(14.3)$ & \\
\hline Partial response & $147.7(2.5)$ & $<0.001$ & $49.8(2.6)$ & $<0.001$ \\
\hline
\end{tabular}

The $p$-value compares those with progressive disease versus those with stable disease and progressive disease versus those with partial response. 
tial surgery and radiotherapy. Ninety patients received adjuvant chemotherapy with temozolomide following radiation \pm temozolomide. Repeat surgical resection was performed in 51 of the patients. The median follow-up time is 118 weeks (Table 1).

Overall, patients were heavily pretreated with approximately $60 \%$ of the patients receiving at least three chemotherapeutic agents before starting tamoxifen. Specifically, patients with transformed low-grade glioma and anaplastic glioma received a median of three agents while GBM patients received two agents prior to initiating tamoxifen. In all cases, patients required an Eastern Cooperative Oncology Group (ECOG) score of 0-2 as per institutional policy to receive systemic therapy.

We observed that approximately $80 \%$ of patients in this heavily pretreated patient population progressed on tamoxifen. A total of 88 patients had repeat surgical resection and $65(73.82 \%)$ of these patients progressed. Utilizing a univariate data analysis, a significant difference in the TTP was seen among those with progressive disease compared to those with at least a stable disease (Table 2).

We also observed that patients on tamoxifen had an overall mOS of 24.9 weeks (range: 1.6 to 346.1 weeks). For those patients who progressed shortly after starting tamoxifen, mOS was similar for GBM (20.5 weeks) and non-GBM (22.7 weeks) patients. However, for patients experiencing stable disease on tamoxifen, the mOS for both GBM (43.3 weeks) and non-GBM (83.2 weeks) patients were improved. For those patients with partial response on tamoxifen, GBM patients were observed having a mOS of 169.4 weeks versus 328.1 weeks for non-GBM patients although numbers were quite small (Table 3).

We were only able to determine MGMT promoter methylation status in 30 patients ( 23 methylated; 7 unmethylated) as many of these patients were treated before MGMT assessment became standard. Patients who were unmethylated were found to have shorter TTP (4.6-9.7 weeks) and mOS (12.3-47.3 weeks) irrespective of pathology. We observed 15 patients with IDH mutation out of a total of 23 patients who were tested. Patients with either IDH1/IDH2 mutation were found to have a relatively longer median TTP of 21.8 weeks and mOS of 175.4 weeks. However, the number of patients with MGMT and IDH status were too small to understand its impact on the response to treatment.

Toxicity was evaluated and with the exception of infre- quent venous thromboembolism, patients universally tolerated tamoxifien with no side effects that could not also be attributed to disease progression.

\section{Discussion}

The management of recurrent high grade glioma remains controversial with no standardized approach validated by large clinical trials. The focus for treatment in these patients is maximization of survival while minimizing toxicity. Current data suggests that median survival after recurrence using a variety of treatment options including re-operation, re-irradiation and systemic therapy varies between 28 to 44 weeks [10].

We retrospectively reviewed 167 heavily pretreated recurrent high-grade glioma patients who were treated with oral tamoxifen as salvage therapy. In this study, we observed that $80 \%$ of GBM and non-GBM patients treated with tamoxifen experienced progression. However, in those patients who had stable disease or partial response, mOS in excess of 40 weeks was observed for GBM and more than 80 weeks for non-GBM patients. On average, these patients were heavily pretreated as tamoxifen was given as salvage therapy after the third or even fourth recurrence. Unfortunately, we were not able to identify any specific clinical characteristics that predicted which patients were more likely to experience stable disease or response.

Overall, our data compares well to the published data. To date, oral tamoxifen used as a single agent has been shown to have mOS greater than 50 weeks for anaplastic astrocytoma and 30 weeks for GBM $[15,16]$. Importantly, no significant toxicities were reported. The key difference between our results and those reported in the literature are that tamoxifen was used in first recurrence on average in these studies whereas patients had multiple treatments before initiating tamoxifen in our study.

The benefit of oral tamoxifen for recurrence is less clear when used in combination with other agents. Three studies combined oral tamoxifen with either temozolomide, procarbazine or carboplatin [17-19]. The mOS was seen to vary between 52 to 67 weeks for recurrent anaplastic glioma and between 23 to 27 weeks for recurrent GBM. No significant toxicities were reported. However, data from three other studies using tamoxifen in combination with concurrent continuous temozolomide, subcutaneous interferon alpha or bortezomib were limited by toxicity and discontinued [20-22]. Two of these discontinued studies employed dosing of up to $100 \mathrm{mg}$

Table 3: Median Survival according to the response to tamoxifen chemotherapy.

\begin{tabular}{|l|l|l|l|l|}
\hline \multirow{2}{*}{ Response } & Median survival in weeks & p-value & GBM $\mathbf{~}=\mathbf{8 1}$ & p-value \\
\cline { 2 - 4 } & Non-GBM $\mathbf{~ n = 8 6}$ & & 22.7 & $<0.001$ \\
\hline Progressive disease & 20.5 & 0.104 & 43.3 & $<0.001$ \\
\hline Stable disease & 83.2 & 0.002 & 169.4 & \\
\hline Partial response & 328.1 & & \\
\hline
\end{tabular}

The $p$-value compares those with progressive disease versus those with stable disease and progressive disease versus those with partial response. 
twice daily in combination with continuous temozolomide or and up to $240 \mathrm{mg}$ daily with subcutaneous interferon alpha. The main toxicities were transaminitis, pancytopenia, herpes zoster, deep vein thrombosis and fatigue, intolerable dizziness and unsteady gait. The third study evaluating tamoxifen in combination with $\mathrm{nf}-\mathrm{kB}$ acting agent borzetomib in 42 patients (12 anaplastic glioma and $30 \mathrm{GBM}$ ) had a mOS of 15-25 weeks but was stopped due to both futility and toxicity [22]. On balance, studies using single-agent tamoxifen or tamoxifen combination therapy for recurrence demonstrated mOS on par or better than those seen historically with general systemic therapy; however, less toxicity was observed using single-agent tamoxifen making it preferable to combination therapy.

This study represents the largest single study assessment using tamoxifen for recurrence in high-grade glioma patients. However, this study is limited by its retrospective nature. In addition, we lack molecular marker data on all patients because this data was not readily available from patients enrolled prior to its use in standard care. Finally, given that this is a chart review, we lack toxicity data graded by Common Terminology Criteria for Adverse Events (CTCAE criteria). To address these weaknesses, we advocate for randomized studies comparing single agent tamoxifen to standard therapies.

Overall, our review helps to describe real world data on oral tamoxifen's use as a single-agent, systemic therapy for recurrence of high grade glioma. Although this is just a retrospective study and we cannot make any actual conclusions on improved survival, this study demonstrates seemingly long survival data for those who are able to achieve stable disease or partial response. Our data suggest that single-agent oral tamoxifen is an appealing target for future randomized trial in the heavily pre-treated patient population of recurrent high grade glioma. We are currently developing such a study at our centre and will be recruiting patients in the foreseeable future.

\section{Compliance with Ethical Standards}

\section{Funding}

No associated funding agency for the study.

\section{Conflict of interest}

Nothing to disclose.

\section{Ethical approval}

Ethics Committee Review approval was not required for this study but all procedures performed were in accordance with the ethical standards of the institutional research committee and with the 1964 Helsinki declaration and its later amendments or comparable ethical standards.

For this type of study, formal consent was not required.

\section{References}

1. Louis DN, Ohgaki H, Wiestler OD, et al. (2016) WHO classification of tumours of the central nervous system. ( $4^{\text {th }}$ edn), IARC, Lyon.

2. Quant EC, Drappatz J, Wen PY (2010) Recurrent high-grade glioma. Curr Treat Options Neurol 12: 321-333.
3. Koshy M, Villano JL, Dolecek TA, et al. (2012) Improved survival time trends for glioblastoma using the SEER 17 population-based registries. J Neurooncol 107: 207-212.

4. Perry JR, Bélanger K, Mason WP, et al. (2010) Phase II trial of continuous dose-intense temozolomide in recurrent malignant glioma: Rescue study. J Clin Oncol 28: 2051-2057.

5. Taal W, Oosterkamp HM, Walenkamp, et al. (2014) Single-agent bevacizumab or lomustine versus a combination of bevacizumab plus lomustine in patients with recurrent glioblastoma (BELOB trial): A randomised controlled phase 2 trial. Lancet Oncol 15: 943-953.

6. Leonard A, Wolff JE (2013) Etoposide improves survival in highgrade glioma: A meta-analysis. Anticancer Res 33: 3307-3315.

7. Friedman HS, Prados MD, Wen PY, et al. (2009) Bevacizumab alone and in combination with irinotecan in recurrent glioblastoma. J Clin Oncol 27: 4733-4740.

8. Brada M, Stenning S, Gabe R, et al. (2010) Temozolomide versus procarbazine, lomustine, and vincristine in recurrent high-grade glioma. J Clin Oncol 28: 4601-4608.

9. Jaeckle KA, Hess KR, Yung WK, et al. (2003) Phase II evaluation of temozolomide and 13-cis-retinoic acid for the treatment of recurrent and progressive malignant glioma: A North American brain tumor consortium study. J Clin Oncol 21: 2305-2311.

10. Huncharek M, Muscat J (1998) Treatment of recurrent high grade astrocytoma; Results of a systematic review of 1,415 patients. Anticancer Res 18: 1303-1311.

11. Myra van Linde ME, Brahm CG, de Witt Hamer PC, et al. (2017) Treatment outcome of patients with recurrent glioblastoma multiforme: A retrospective multicenter analysis. J Neurooncol 135: 183-192.

12. Fisher B, Costantino J, Redmond C, et al. (1989) A randomized clinical trial evaluating tamoxifen in the treatment of patients with node-negative breast cancer who have estrogen-receptor-positive tumors. N Eng J Med 320: 479-484.

13. O'Brian CA, Solomon DH, Liskamp RM, et al. (1985) Inhibition of protein kinase C by tamoxifen. Cancer Res 45: 2462-2465.

14. Todorova VK, Kaufmann Y, Luo S, et al. (2011) Tamoxifen and raloxifene suppress the proliferation of estrogen receptor-negative cells through inhibition of glutamine uptake. Cancer Chemother Pharmacol 67: 285-291.

15. Couldwell WT, Hinton DR, Surnock AA, et al. (1996) Treatment of recurrent malignant gliomas with chronic oral high-dose tamoxifen. Clin Cancer Res 2: 619-622.

16. Chamberlain MC, Kormanik PA (1999) Salvage chemotherapy with tamoxifen for recurrent anaplastic astrocytomas. Arch Neurol 56: 703-708.

17. DI Cristofori A, Carrabba G, Lanfranchi G, et al. (2013) Continuous tamoxifen and dose-dense temozolomide in recurrent glioblastoma. Anticancer Res 33: 3383-3389.

18. Brandes AA, Ermani M, Turazzi S, et al. (1999) Procarbazine and high-dose tamoxifen as a second-line regimen in recurrent highgrade gliomas: A phase II study. J Clin Oncol 17: 645-650.

19. Tang P, Roldan G, Brasher PM, et al. (2006) A phase II study of carboplatin and chronic high-dose tamoxifen in patients with recurrent malignant glioma. J Neurooncol 78: 311-316.

20. Chang SM, Barker FG 2nd, Huhn SL, et al. (1998) High dose oral tamoxifen and subcutaneous interferon alpha-2a for recurrent glioma. J Neurooncol 37: 169-176. 
21. Spence AM, Peterson RA, Scharnhorst JD, et al. (2004) Phase II study of concurrent continuous Temozolomide (TMZ) and Tamoxifen (TMX) for recurrent malignant astrocytic gliomas. J Neurooncol 70: 91-95.
22. Odia Y, Kreisl TN, Aregawi D, et al. (2015) A phase II trial of tamoxifen and bortezomib in patients with recurrent malignant gliomas. J Neurooncol 125: 191-195. 